\title{
Creation and luminescence of size-selected gold nanorods $\dagger$
}

\author{
Y. Chen, ${ }^{* a}$ Y. Zhang, ${ }^{a}$ D. J. S. Birch ${ }^{a}$ and A. S. Barnard ${ }^{b}$ \\ Received 10th February 2012, Accepted 11th June 2012 \\ DOI: $10.1039 / \mathrm{c} 2 \mathrm{nr} 30324 \mathrm{~h}$
}

Fluorescent metal nanoparticles have attracted great interest in recent years for their unique properties and potential applications. Their optical behaviour depends not only on size but also on shape, and will only be useful if the morphology is stable. In this work, we produce stable size-selected gold nanorods (aspect ratio 1-2) using a size-selected cluster source and correlate their luminescence behaviour with the particle shape. Thermodynamic modelling is used to predict the preferred aspect ratio of 1.5 , in agreement with the observations, and confirms that the double-icosahedron observed in experiments is significantly lower in energy than the alternatives. Using these samples a fluorescence lifetime imaging microscopy study observed two photon luminescence from nanoparticle arrays and a fast decay process $(<100$ ps luminescence lifetime), which are similar to those found from ligand stabilized gold nanorods under the same measurement conditions, indicating that a surface plasmon enhanced two-photon excitation process is still active at these small sizes. By further reducing the nanoparticle size, this approach has the potential to investigate size-dependent luminescence behaviour at smaller sizes than has been possible before.

The photoluminescence from bulk gold was first reported by Mooradian in 1969 (ref. 1) with a quantum yield in the order of $10^{-10}$. An increase in the intensity of multiphoton excited luminescence was found later from a rough gold surface ${ }^{2}$ as a result of local-field enhancement by surface plasmon resonances. This kind of enhancement has also been proposed for the observed luminescence from gold nanostructures, where the luminescence efficiency can increase by up to six orders of magnitude. ${ }^{3}$

The last decade has seen an increased interest in luminescent gold nanoparticles because of their unique optical properties and potential in biological sensing, imaging and nanophotonic devices. ${ }^{4-7}$ Among nanoparticles with different structures, gold nanorods have proven to be the most flexible structure because of their shape-dependent optoelectronic properties. ${ }^{\mathbf{8} 9}$ In addition to strong two-photon luminescence, ${ }^{\mathbf{1 0 , 1 1}}$ gold nanorods have low toxicity and the capability of conjugating to biomolecules, thus becoming promising candidates for biological sensing and imaging. ${ }^{12-21}$ Previously we demonstrated that by incorporating gold nanorods into cells, their fast fluorescence decay time for

${ }^{a}$ Photophysics Group, Centre for Molecular Nanometrology, Department of Physics, SUPA, University of Strathclyde, John Anderson Building, 107 Rottenrow, Glasgow, G4 ONG, UK.E-mail: y.chen@strath.ac.uk; Fax: +44 (0)141 5522891; Tel: + 44 (0)141 5483087

${ }^{b}$ Virtual Nanoscience Laboratory, CSIRO Materials Science \& Engineering, Parkville, 3052, Australia. E-mail: amanda.barnrd@csiro. au; Fax: +61-3-9962-7145; Tel: +61-4-9962-7356

$\uparrow$ Electronic supplementary information (ESI) available: Electron micrographs of gold nanorods on an amorphous carbon TEM support, using scanning transmission electron microscopy with a high angle annular dark field detector, and representative intensity 3D plots. See DOI: $10.1039 / \mathrm{c} 2 \mathrm{nr} 30324 \mathrm{~h}$ two-photon luminescence could be used efficiently in fluorescence lifetime imaging microscopy (FLIM). ${ }^{15}$ Moreover, the enhanced energy transfer between a DNA dye, DAPI, and gold nanorods under two-photon excitation was observed, suggesting their potential not only in Förster resonance energy transfer (FRET) imaging, but also in fluorescence lifetime-based intracellular sensing. ${ }^{16}$

The optical properties of metal nanoparticles depend not only on size but also on shape, which is why the anisotropy of gold nanorods is so important. Most of the work published to date has used gold nanorods synthesized via a seed mediated growth process ${ }^{22}$ as a greater level of control is assumed. The nanorods are typically prepared using the surfactant cetyltrimethylammonium bromide $(\mathrm{CTAB})$ to produce an average length in the range of a few tens of nanometres (e.g., about $40 \mathrm{~nm}$ for nanorods of aspect ratio about 3). These nanorods are relatively large, and the prospect of producing size-selected nanorods on the scale of $10 \mathrm{~nm}$ is attractive, since the local-field enhancement would be even greater. To date, however, seed mediated growth suffers from some (seemingly) insurmountable challenges at such small sizes.

Here we report the investigation of the formation of size selected gold nanoparticles and their thermodynamically stable shapes. Luminescence was studied using fluorescence lifetime imaging microscopy and compared with colloidal gold nanorods. Compared to colloidal nanoparticles synthesized through a chemical process (such as seed mediated growth), nanoparticles produced using this method have a well-controlled size $(5 \%$ resolution) which is tuneable from a few atoms up to hundreds of thousands of atoms (corresponding to sub-nanometre to a few 

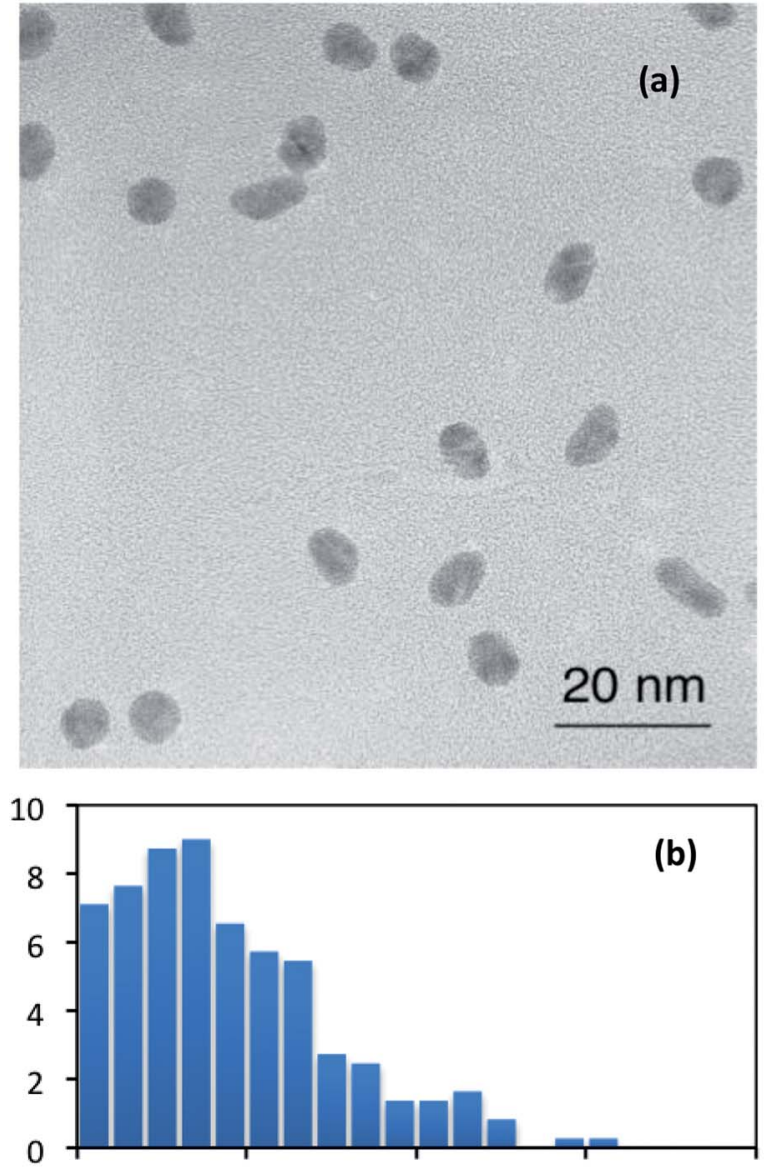

1

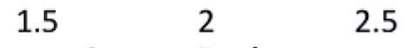

Aspect Ratio
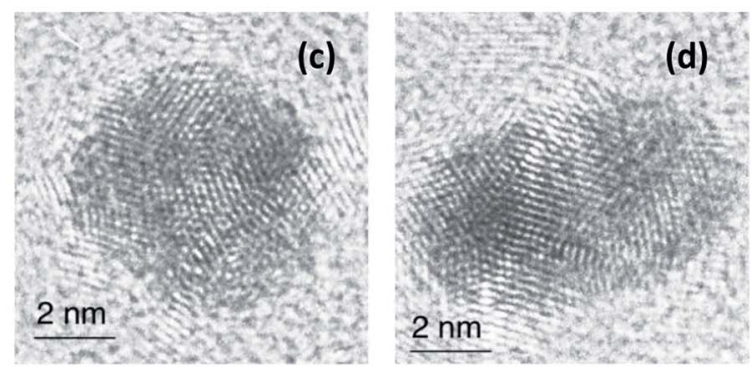

Fig. 1 TEM micrographs taken from $\mathrm{Au}_{8860}$ nanoparticles (a), and distributions of the aspect ratio for nanoparticles grown under optimum He flow rate (b). A single nanoparticle with a quasi-spherical shape (c) and an elongated shape (d).

nanometres in dimension). This facilitates a study on the evolution of optical properties, from the molecular characteristics of small nanoparticles to the surface plasmon dominated behaviour of large nanoparticles. In addition to size-control, nanoparticles thus formed are free of surfactant, which allows for a study on the influence of surface chemical states on the optical behaviour.

Since the intrinsic luminescence depends heavily on the selectivity (size) and dynamical shape (nanorod anisotropy), we will begin by outlining the synthesis methods and providing a theoretical analysis of the stability under the characterisation conditions. This is followed by a discussion of the optical properties, based on complementary techniques.
In this work, we used a size-selected cluster source to create nanoparticles consisting of a specific number of atoms, and with the desired dimension less than $10 \mathrm{~nm}$. The size-selected cluster source employs a novel mass-selection technique based on the time-of-flight principle that has been implemented into a radio frequency magnetron sputtering and gas condensation processes. ${ }^{23,24}$ This method has been described in detail elsewhere, and has the advantage of simultaneously identifying the size with atomic level precision (via mass spectrometry) and providing the required degree of selectivity. In the first instance, gold nanoparticles with 8860 atoms were selected and deposited on a TEM grid with a kinetic energy of $500 \mathrm{eV}$. Fig. 1(a) shows a typical TEM micrograph taken at $200 \mathrm{keV}$ using a Tecnai F20 transmission electron microscopy (TEM) from FEI. As indicated in the typical image, well-separated nanoparticles were observed scattered on the support.

Particles were found with various shapes, ranging from near spherical to elongated. The anisotropy of the particles is characterised by measuring the aspect ratio of the individual particles, and Fig. 1(b) displays the distribution of the aspect ratio. We can see here that majority of particles have slightly distorted, non-spherical shape with an aspect ratio distribution peaking at about 1.3. The particle number reduces with the increase in the aspect ratio; nevertheless, elongated particles with aspect ratio larger than 2 are observed. However, upon closer inspection, high-resolution micrographs reveal icosahedral structures, as displayed in Fig. 1(c) and (d), which is consistent with the thermodynamically preferred shape of quasi-spherical gold
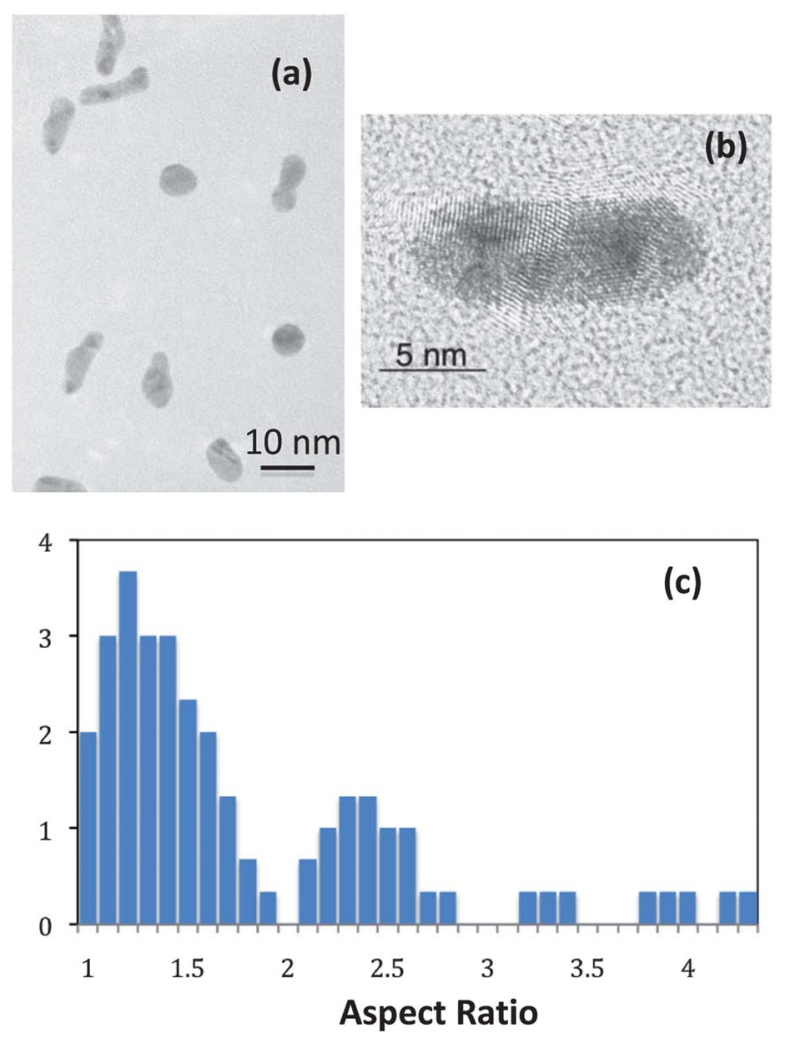

Fig. 2 TEM micrographs taken from $\mathrm{Au}_{15000}$ nanoparticles (a) and single nanoparticles with aspect ratio of 3.3 (b); distribution of aspect ratio for $\mathrm{Au}_{15000}$ nanoparticles under optimum He flow rate (c). 
nanoparticles of this size, ${ }^{25}$ but inconsistent with the structure observed in gold nanorods produced via seed mediated methods. Although predictions have been made regarding the preferred orientation and aspect ratio of $\mathrm{fcc}^{26}$ and decahedral ${ }^{27}$ gold nanorods, no attempt has been made to integrate dimensional anisotropy with the size dependent phase diagram of gold, ${ }^{25}$ so it is unclear if these anisotropic icosahedral structures will be thermodynamically stable.

To verify the reproducibility of these results, and to study the effect of size on the particle shape, nanoparticles consisting of 15000 gold atoms were also prepared using the same methods described briefly above. A TEM image, Fig. 2(a), shows that these larger nanoparticles are also well-separated, and nanorods with multi-domain structures are clearly visible. Particles with close to a triangular shape are also observed, which suggests formation of nanoparticles through collision and coalescence between intermediate sized nanoparticles. The aggregate remains an elongated structure due to a limited sintering as the energy generated after initial coalescence is not high enough to drive the mass diffusion to reach an overall low energy structure. ${ }^{28}$ This implies contributions from two complementary growth processes: coagulation and successive atom assembly (addition). While the first process manifests itself in the formation of largely distorted or elongated nanoparticles, the second process is responsible for the formation of quasi-spherical nanoparticles. Aspect ratio analysis, Fig. 2(c), shows similar distributions as those found for $\mathrm{Au}_{8860}$, although more rod-like nanoparticles were found. Fig. 2(b) shows one example of these rod-like nanoparticles with an aspect ratio of 3.3 .

It is clear that most of the nanoparticles created in this work have an asymmetric shape with aspect ratios between 1 and 2 (peaked at 1.3-1.4), but it is unclear if this is a kinetic or thermodynamic effect. Icosahedral structures are kinetically stable (even at larger sizes) due to a superior rate of coarsening, ${ }^{29}$ but they will only be of use in nanophotonic applications if they are thermodynamically stable under the relevant conditions.

To address this issue we modelled the temperature dependent free energy $(G)$ of a set of possible nanoparticle geometries, using an established shape-dependent thermodynamic model ${ }^{30}$ and parameters obtained from relativistic density functional theory. ${ }^{31}$ The shapes, shown in Fig. 3, are all enclosed by low index surfaces, with different degrees and configurations of (111) twinning. The free energy of $110 \mathrm{~K}$ is provided as a function of the aspect ratio (with the volume conserved) in Fig. 4(a) and (b) for the 8860 atom and 15000 atom structures, respectively. In both cases we see that the icosahedral shape observed in our experiments (Fig. 1(a)) is significantly lower in energy than the alternatives (as predicted by the phase diagram), and that the energetically preferred aspect ratio is 1.5 , corresponding to a double icosahedron, ${ }^{32}$ as displayed in Fig. 1(c) and 3. This particular shape is lower in energy than the simple (quasi-zero dimensional) icosahedron, and lower in energy than alternatives with higher aspect ratios. Based on these results (and the fact that our formation conditions are locally homogeneous, suggesting there is no systematic anisotropy during kinetic coarsening), ${ }^{29} \mathrm{we}$ can conclude that, among the nanoclusters of different aspect raios, double icosahedra (of aspect ratio of $\sim 1.5$ ) are expected to be thermodynamically stable during investigation of their optical properties (and future application), irrespective of formation mechanisms.

Having established a reliable way of producing size-selected nanorods, that will be stable under a large range of thermal conditions, we turn our attention to the luminescent properties. The 15000 atom Au nanoparticles were deposited onto a glass slide to create sub-monolayer array, and covered with a cover slip. Fluorescence lifetime imaging microscopy (FLIM) was performed using a confocal microscope (LSM 510, Carl Zeiss) equipped with a time-correlated single photon counting (TCSPC) module (SPC-830, Becker \& Hickl GmbH), and a femtosecond Ti:Sapphire laser (Chameleon, Coherent) was tuned to $750 \mathrm{~nm}$. The laser pulse has a repetition rate of $80 \mathrm{MHz}$ and a duration of less than $200 \mathrm{fs}$. Emission was collected by a $40 \times$ oil-immersion objective $($ N.A. $=1.3$ ) and a bandpass filter with a transmission window from $500 \mathrm{~nm}$ to $550 \mathrm{~nm}$ (the highest fluorescence intensity measured in this range). The nonlinear property of the luminescence was studied by measuring the
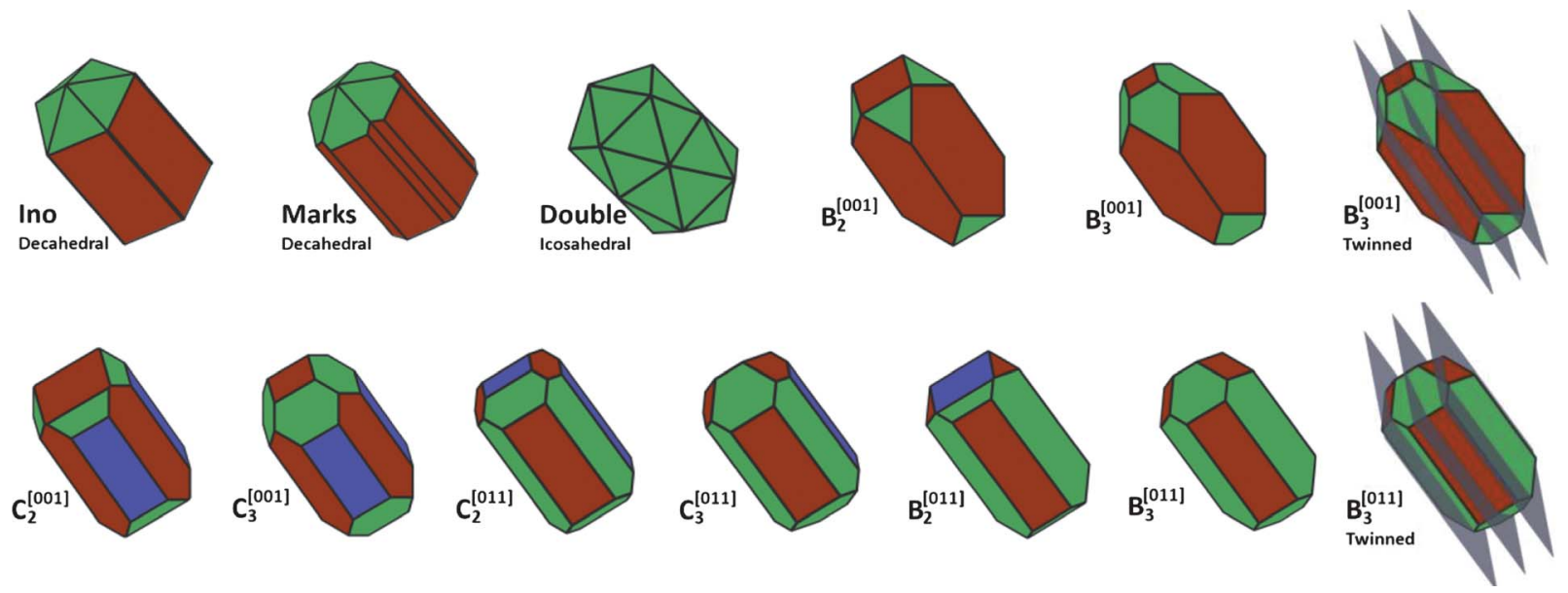

Fig. 3 Set of sample shapes investigated using theoretical modelling. The naming convention is consistent with ref. 26, where the superscript denotes the orientation of the principle axis. The (100) facets are shown in red, the (111) facets are shown in green, and the (110) facets are shown blue. Twin planes in fcc structures are shown in grey. The free energy for each of these shapes and structures has been calculated analytically (based on quantum mechanical simulations) and is shown in Fig. 4. 

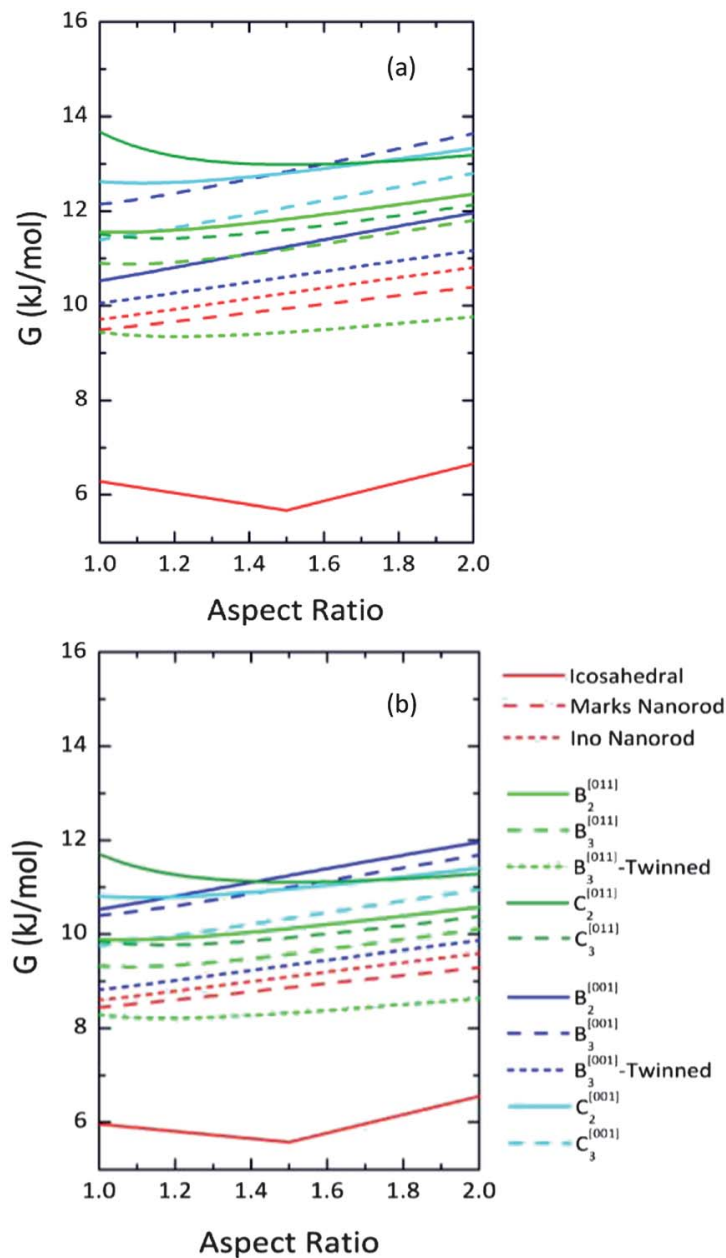

Fig. 4 Calculated free energy of formation $(G)$ at $110 \mathrm{~K}$ for the gold nanorods shown in Fig. 3, for (a) $\mathrm{Au}_{8860}$, and (b) $\mathrm{Au}_{15000}$. The volume is conserved at all aspect ratios, and the double-icosahedral nanorod is the lowest energy shape among those considered (at the size and temperature under consideration). The double-icosahedron is preferred over alternatives with a higher degree of dimensional anisotropy (1-D), or a single icosahedron (0-D).

luminescence intensity as a function of excitation power ranging from 1.2 to $10.2 \mathrm{~mW}$ and shown to be two-photon in origin.

Fig. 5(a) shows a luminescence intensity image, where the bright spots represent the emission from individual gold nanoparticles. We have analyzed different imaging pixels and display a typical result in Fig. 5(b), in comparison with that from chemically synthesized gold nanorods and 4'-6-diamidino-2phenylindole (DAPI) under typical two-photon excitation processes and the same experimental conditions (plotted for reference). A near quadratic dependence of the signal intensity on the input power, nearly identical to colloidal gold nanorods and close to DAPI, Fig. 5(c), suggests that the luminescence of nanoparticles from both synthetic sources originates primarily from a two-photon excitation process. Measurement in a cuvette confirmed the two-photon behaviour for colloidal gold nanorods, but we have noticed that microscope measurements often provide a lower value, possibly due to saturation of the low density luminescence site. These measurements confirm that our gold nanorods, produced using the size-selected cluster source, have a similar optical behaviour to those produced using the traditional seed-mediated growth methods, and will therefore be suitable for the same types of applications.

The corresponding lifetime image, Fig. 5(b), with different colour codes representing different lifetime scales, also correlates well with its intensity counterpart. The luminescence lifetime of each pixel is obtained by applying a single or multi-exponential fit; in this case a single exponential model was used. The densely packed orange spots in Fig. 5(b) have a decay time shorter than

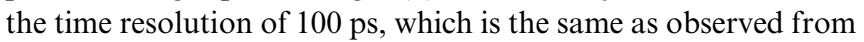
CTAB stabilized gold nanorods under the same experimental conditions. ${ }^{15}$ Moreover, the sample in the absence of gold nanorods showed no fluorescence with a decay time faster than the 100 ps as displayed by the gold nanorods. These results confirm that our gas-cluster source nanorods have comparable optical properties to seed-mediated nanorods. ${ }^{33-35}$ The twophoton luminescence of seed-mediated gold nanorods is dominated by surface plasmon resonance. With the decrease of size, the surface plasmon resonance becomes broadened and weak due to electron-surface scattering and size dependent extinction cross-section. ${ }^{36}$ Small nanoparticles, with a size below $2 \mathrm{~nm}$, no longer possess plasmon resonance, but have molecule-like luminescence features with longer lifetimes. ${ }^{37}$ Because the absorption is too weak to be readily measurable, and to examine the possible influence of a surface plasmon, we calculated the extinction spectrum of our gas-cluster source nanorods using Mie theory [S2†]. The extinction spectrum of $\mathrm{Au}_{15000}$, Fig. 6, shows a broad surface plasmon absorption ranging over 460 $800 \mathrm{~nm}$, overlapped with the excitation at $750 \mathrm{~nm}$. This combines with similar two-photon luminescence characteristics to those of
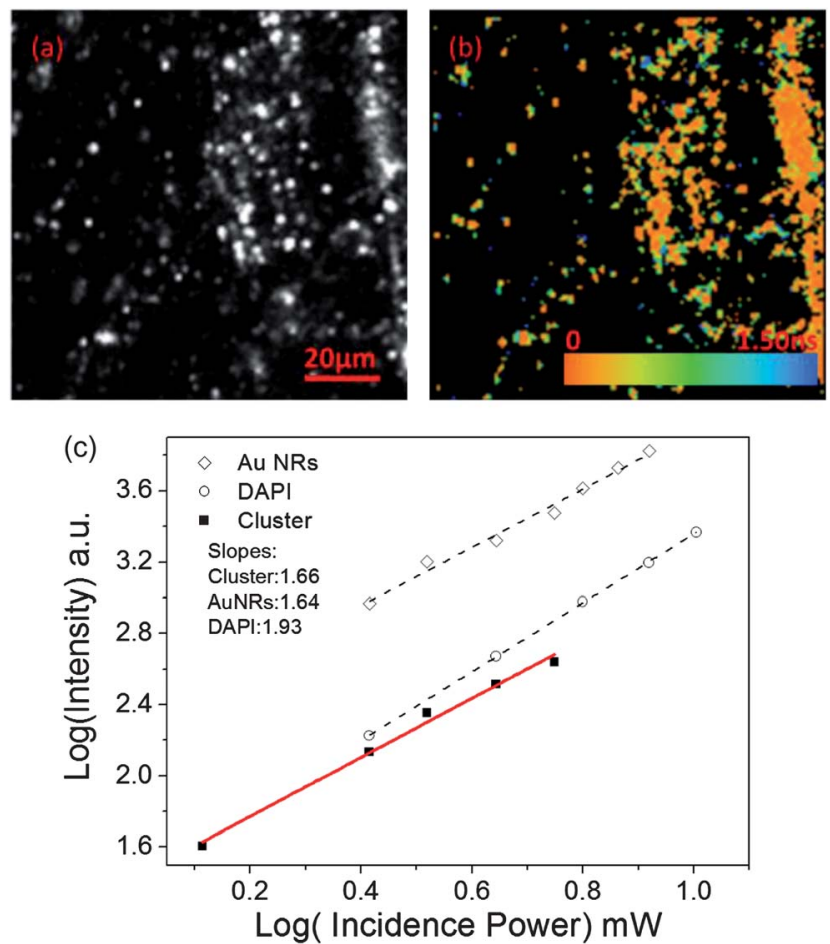

Fig. 5 (a) Luminescence intensity and (b) lifetime images of gold nanoparticles $\left(\mathrm{Au}_{15000}\right)$; (c) dependence of luminescence intensity on incidence laser power (in log scale). 


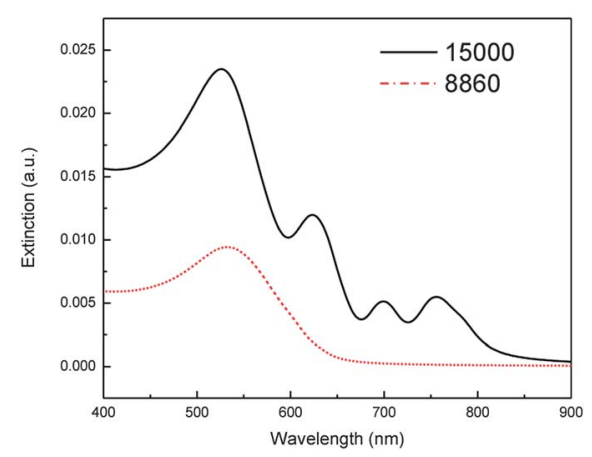

Fig. 6 Calculated extinction spectra of $\mathrm{Au}_{15000}$ and $\mathrm{Au}_{8860}$ nanocluster ensembles with aspect ratio distributions as shown in Fig. $1 \mathrm{~b}$ and 2c.

the colloidal nanorods observed in the experiment, suggesting that both luminescences arise from a similar process. Luminescence intensity and luminescence lifetime are arguably the most important defining characteristics of nanoparticles for use in biological sensing, imaging and nanophotonic devices. Seedmediated gold nanorods have demonstrated potentials in biomedical imaging and intra-cellular sensing, in combination with multi-photon microscopy and resonance energy transfer based lifetime microscopy. Here, these optical properties are incorporated with low toxicity, as they are free of CTAB,${ }^{38}$ and a small size of a few nanometers (comparable to proteins), suggesting that these gold nanorods are highly promising to serve as a fluorescent probe in biomedical applications.

Occasional bluish patches/pixels in Fig. 5(b) with lifetimes longer than nanoseconds are noticeable. They can be easily distinguished from the emissions from gold nanoparticles due to the significant difference in the lifetimes under two-photon excitation, and probably due to contaminants. In addition, these contamination spots show strong luminescence under single photon excitation, while signals from gold nanoparticles are below the detection limit. Luminescence is also observed from gold nanoparticles with 8860 atoms. But the intensity is much lower, about 20 times less than that from $\mathrm{Au}_{15000}$. This is not unexpected considering a reduced number of nanoparticles of large aspect ratio (rod-like nanoparticles) (Fig. 1(d)), resulting in a much weaker surface plasmon resonance at excitation wavelengths and therefore low luminescence, also suggested by the calculated extinction spectrum (Fig. 6).

A keen eye will notice from both intensity and lifetime images that the fluorescent gold nanoparticles are not always uniformly distributed, Fig. 5. Fig. 7 shows intensity and lifetime images
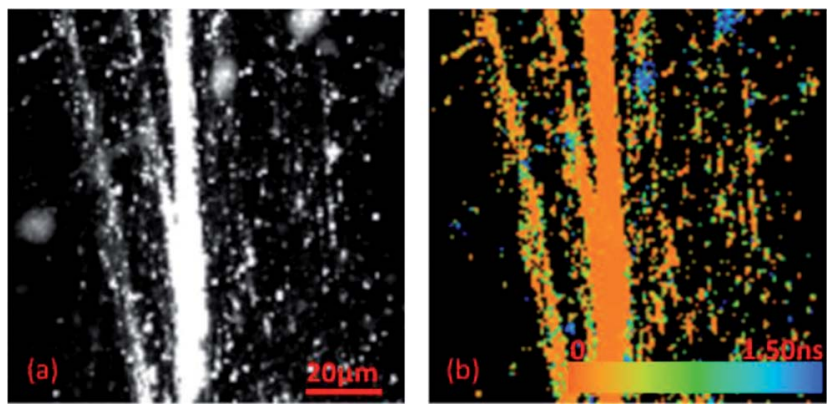

Fig. 7 (a) Luminescence intensity and (b) lifetime images showing gold nanoparticles $\left(\mathrm{Au}_{15000}\right)$ assembled along trenches on a glass slide. taken from an area of the surface decorated with numerous trenches. Bright lines on both images indicate aggregation of nanoparticles in these trenches, whereas the relatively dark areas between the trenches suggest a low coverage of nanoparticles. This is a result of nanoparticle diffusion after deposition at room temperature, and the nanoparticles being pinned at low energy defect sites. This distinction may seem trivial at first, but actually suggests the possibility of creating luminescent nanostructures through substrate surface patterning, which is another feature often attributed to seed-mediated nanoparticles.

In summary, we have created luminescent gold nanoparticles using a size selected cluster source, the majority of which have asymmetric thermodynamically stable shapes. The TPL process and short lifetime are confirmed to be similar to those found from CTAB stabilized gold nanorods under the same measurement conditions, indicating that a surface plasmon dominated two-photon excitation process is still active at sizes much smaller than those typically produced using wet chemical methods. A microscopic study also suggests the tantalizing possibility of creating luminescent nanostructures through assembling gold nanoparticles at patterned surface features. In contrast to large Au nanoparticles, small metal nanoparticles of sizes comparable to the Fermi wavelength of electrons ( $c a .0 .7 \mathrm{~nm}$ ) no longer possess plasmon resonance, ${ }^{39}$ but have molecule-like properties including size-dependent luminescence ${ }^{40-42}$ and discrete electronic states. ${ }^{4-45}$ By further reducing the nanoparticle size, this approach provides the possibility to investigate size dependent luminescence behaviour and the evolution of surface plasmon resonance, especially in combination with single particle spectroscopy and microscopy. ${ }^{46}$

\section{Acknowledgements}

We thank Dr N. Lidgi-Guigui for the assistance in sample preparation and Prof. R. E. Palmer for supporting this work. This work was sponsored by an EPSRC Science and Innovation Award and the ARC under Discovery Grant DP0986752.

\section{Notes and references}

1 A. Mooradian, Phys. Rev. Lett., 1969, 22, 185.

2 G. T. Boyd, A. H. Yu and Y. R. Shen, Phys. Rev. B: Condens. Matter Mater. Phys., 1986, 33, 7923.

3 M. B. Mohamed, V. Volkov, S. Link and M. A. El-Sayed, Chem. Phys. Lett., 2000, 317, 517.

4 X. Huang, S. Neretina and M. A. El-sayed, Adv. Mater, 2009, 21, 4880.

5 R. Sardar, A. M. Funston, P. Mulvaney and R. W. Murray, Langmuir, 2009, 25, 13840.

6 Y. Chen, J. A. Preece and R. E. Palmer, Ann. N. Y. Acad. Sci., 2008, 1130, 201.

7 P. Zijlstra, J. W. M. Chon and M. Gu, Nature, 2009, 459, 410.

8 J. Perez-Juste, I. Pastoriza-Samtos, L. M. Liz-Marzan and P. Mulvaney, Coord. Chem. Rev., 2005, 249, 1870.

9 M. Grzelezak, J. Perez-Juste, P. Mulvaney and L. M. Liz-Marzan, Chem. Soc. Rev., 2008, 37, 1783.

10 H. Wang, T. B. Huff, D. A. Zweifel, W. He, P. S. Low, A. Wei and J. Cheng, Proc. Natl. Acad. Sci. U. S. A., 2005, 102, 15752.

11 K. Imura, T. Nagahara and H. Okamoto, J. Phys. Chem., 2005, 109, 13214.

12 X. Qian, X. H. Peng, D. O. Ansari, Q. Y. Goen, G. Z. Chen, D. M. Shin, L. Yang, A. M. Young, M. D. Wang and S. Nie, Nat. Biotechnol., 2008, 26, 83. 
13 C. J. Orendorff, L. Gearheart, N. R. Jana and C. J. Murphy, Phys. Chem. Chem. Phys., 2006, 8, 165.

14 X. G. Hu, W. L. Cheng, T. Wang, Y. L. Wang, E. K. Wang and S. J. Dong, J. Phys. Chem. B, 2005, 109, 19385.

15 Y. Zhang, J. Yu, D. J. S. Birch and Y. Chen, J. Biomed. Opt., 2010, 15, 020504 .

16 Y. Zhang, D. J. S. Birch and Y. Chen, Appl. Phys. Lett., 2011, 99 103701 .

17 T. B. Hull, L. Tong, Y. Zhao, M. N. Hansen, J. X. Cheng and A. Wei, Nanomedicine, 2007, 2, 125.

18 N. J. Durr, T. Larson, D. K. Smith, B. A. Korgel, K. Sokolov and A. Ben-Yakar, Nano Lett., 2007, 7, 941.

19 A. Broioude, X. C. Jiang and M. P. Pileni, J. Phys. Chem. B, 2005, 109, 13138

20 G. W. Lu, L. Hou, T. Y. Zhang, W. Q. Li, J. Liu, P. Perriat and Q. H. Gong, J. Phys. Chem. C, 2011, 115, 22877.

21 C. Burda, X. Chen, R. Narayanan and M. A. El-sayed, Chem. Rev., $2005, \mathbf{1 0 5}, 1025$

22 C. J. Murphy, T. K. Sau, A. M. Gole, C. J. Orendorff, J. Gao, L. Gou, S. E. Hunyadi and T. Li, J. Phys. Chem., 2005, 109, 13875.

23 M. Di Vece, N. P. Young, Z. Li, Y. Chen and R. E. Palmer, Small, 2006, 2, 1270

24 R. E. Palmer, S. Pratontep and H.-G. Boyen, Nat. Mater., 2003, 2, 443.

25 A. S. Barnard, N. Young, A. I. Kirkland, M. A. van Huis and H. Xu, ACS Nano, 2009, 3, 1431.

26 A. S. Barnard and L. A. Curtiss, J. Mater. Chem., 2007, 17, 3315.

27 A. S. Barnard, J. Phys. Chem. C, 2008, 112, 1385.

28 Y. Chen, R. E. Palmer and J. P. Wilcoxon, Langmuir, 2006, 22, 2851.

29 A. S. Barnard and Y. Chen, J. Mater. Chem., 2011, 21, 12239.

30 A. S. Barnard, J. Phys. Chem. B, 2006, 110, 24498.
31 A. S. Barnard, X. M. Lin and L. A. Curtiss, J. Phys. Chem. B, 2005, 109, 24465.

32 H. Hofmeister, Z. Kristallogr., 2009, 224, 528.

33 T. B. Huff, M. N. Hansen, Y. Zhao, J. Cheng and A. Wei, Nanomedicine, 2007, 2, 125.

34 O. P. Varnavski, M. B. Mohamed, M. A. El-Sayed and T. Goodson III, J. Phys. Chem. B, 2003, 107, 3101.

35 P. Biagioni, M. Celebrano, M. Savoini, G. Grancini, D. Brida, S. Mátéfi-Tempfli, M. Mátéfi-Tempfli, L. Duò, B. Hecht, G. Cerullo and M. Finazzi, Phys. Rev. B: Condens. Matter Mater. Phys., 2009, 80, 045411.

36 G. V. Hartland, Chem. Rev., 2011, 111, 3858.

37 J. Xie, Y. Zheng and J. Y. Ying, J. Am. Chem. Soc., 2009, 131, 888.

38 Y. Zhang, D. Xu, W. Li, J. Yu and Y. Chen, J. Nanomater., 2012, 2012, 375496

39 S. Link and M. A. El-Sayed, Annu. Rev. Phys. Chem., 2003, 54, 331.

40 C. C. Huang, Z. Yang, K. H. Lee and H. T. Chang, Angew. Chem., $2007,46,6824$

41 J. Zheng, C. W. Zhang and R. M. Dickson, Phys. Rev. Lett., 2004, 93, 077402.

42 N. Schaeffer, B. Tan, C. Dickinson, M. J. Rosseinsky, A. Laromaine, D. W. McComb, M. M. Stevens, Y. Wang, L. Petit, C. Barentin, D. G. Spiller, A. I. Cooper and R. Levy, Chem. Commun., 2008, 3986.

43 S. Chen, R. S. Ingram, M. J. Hostetler, J. J. Pietron, R. W. Murray, T. G. Schaaff, J. T. Khoury, M. M. Alvarez and R. L. Whetten, Science, 1998, 280, 2098.

44 P. Apell, R. Monreal and S. Lundqvist, Phys. Scr., 1988, 38, 174.

45 Z. Y. Lin, R. P. F. Kanters and D. M. P. Mingos, Inorg. Chem., 1991, 30, 91.

46 P. Zijlstra and M. Orrit, Rep. Prog. Phys., 2011, 74, 106401. 